\title{
Cobalt-60 Source Loading: Necessity of A Rigid Protocol
}

\author{
Ravi Kant ${ }^{1}$, Geomcy George ${ }^{2 *}$, Shekhar Dwivedi ${ }^{1}$, Mahajan $\mathrm{MK}^{1}$, Shahid $\mathrm{KM}^{1}$ and Yadvinder $\mathrm{S}^{1}$ \\ ${ }^{1}$ Advanced Cancer Diagnostic, Treatment and Research Centre, Bathinda, India \\ ${ }^{2}$ Believers Church Medical College Hospital, Thiruvalla, India
}

Submission: : July 01, 2019; Published: July 25, 2019

"Correspondence Author: Geomcy George, Department of Radiation Oncology, Believers Church Medical College Hospital, St. Thomas Nagar, P.O. Box-31, Kuttapuzha, Thiruvalla, Kerala, India

Abstract

Aim: To look if calculating the gantry angle position helps in source loading

Materials and Methods: The study was conducted in Advanced Cancer Diagnostic, Treatment and research Centre- Bathinda, India at the time of source loading in the Bhabhatron - II TAW teletherapy unit. There is no rigid protocol for calculating the angle in which source loading needs to be done. The study looks at various gantry angle positions in which source loading was attempted and failed and describes how to calculate the position in which source loading needs to be done; based on which successful loading was done.

Result: Calculating the gantry angle position helps reduce source loading errors.

Conclusion: By following a rigid protocol, the number of trials undertaken for the cobalt- 60 source loading could be reduced, thus limiting the radiation exposure to all involved.

Keywords: Bhabhatron-II; Cobalt-60; Source loading

\section{Introduction}

Telecobalt units are widely used in developing countries for cancer treatment and are preferred over medical linear accelerators because of:

i) Low cost,

ii) Low maintenance cost,

iii) Lower power requirements, and

iv) Less down time [1]. Bhabha Atomic Research Centre, Mumbai, India has designed and developed a prototype telecobalt unit, which has been named Bhabhatron-I [2]. After receiving feedback on the operations of this unit, a modified model was developed, Bhabhatron-II

Cobalt-60 source loading is a routinely practiced procedure for more than six decades now. This procedure involves a close co-operation between Service Engineer, Medical Physicist, Radiation Therapy Technologist and radiation oncologist. For a smooth operation and reasonably low radiation exposure to the personnel, a workable and time-tested protocol might be more beneficial than an experience based on 'hit and trial' method.
The present study is an attempt to that context evolved during the experience of various problems faced during Cobalt-60 source loading in the Bhabhatron-II-TAW teletherapy unit at Advanced Cancer Diagnostic, Treatment and Research Centre- Bathinda.

\section{Material and Methods}

The study was conducted in Advanced Cancer Diagnostic, Treatment and research Centre- Bathinda, India at the time of source loading in the Bhabhatron - II TAW teletherapy unit. There is no rigid protocol for calculating the angle in which source loading needs to be done. The room preparation was done before source loading. The surface of the floor was flat. Gamma zone monitor in the room was in working condition. There was proper lightening in the room. The various acceptance tests for the treatment unit including electrical and mechanical of the machine were completed. Usual protocols have been defined for the teletherapy unit having the swivel function of the head of the gantry which facilitates rotation of the Gantry head to face towards the floor. Bhabhatron-II unit is not having swivel function in its Gantry Head necessitating for an alternate solution. The Source flask was shifted into the telecobalt room 


\section{Cancer Therapy \& Oncology International Journal}

by hydraulic trolley. Alignment with head of the Gantry was achieved. The gantry angle for source loading was preferred in anti-clockwise direction as the position in clockwise direction interfered with the electrical connections of the couch.

\section{Results}

The Gantry angles from serial no. 1 to 3 were randomly selected by the engineers while the $4^{\text {th }}$ one was calculated as shown in table 1 and detailed in figure 1 . The selection of the angle of 230.10 was calculated using trigonometry and calculus as below:

Flask source drawer center to floor distance $(C G)=68.6 \mathrm{~cm}$

Gantry source drawer $\left(\mathrm{G} \theta=270^{\circ}\right)$ to floor distance $(\mathrm{BF})$ $=120 \mathrm{~cm}$, Gantry rotation center (axis) to floor distance $\mathrm{AH}=120 \mathrm{~cm}$,

Table 1: Showing the details of various attempts undertaken to load the source. Attempt number 4 (with *) was the derived one.

\begin{tabular}{|c|c|c|c|c|c|}
\hline S. No. & Gantry $\boldsymbol{\theta}$ & Couch $\boldsymbol{\theta}$ & Collimator $\boldsymbol{\theta}$ & Jaw Openings & Result \\
\hline 1. & 228 & $90^{\circ}$ & $0^{\circ}$ & $0 \mathrm{X}(0+0.1)$ & not aligned \\
\hline 2. & 229.3 & $90^{\circ}$ & $0^{\circ}$ & $0 \mathrm{X}(0+0.1)$ & not aligned \\
\hline 3. & 231.5 & $90^{\circ}$ & $0^{\circ}$ & $0 \mathrm{X}(0+0.1)$ & not aligned \\
\hline $4 .^{*}$ & 230.1 & $90^{\circ}$ & $0^{\circ}$ & $0 \mathrm{X}(0+0.1)$ & Aligned, source loaded \\
\hline
\end{tabular}

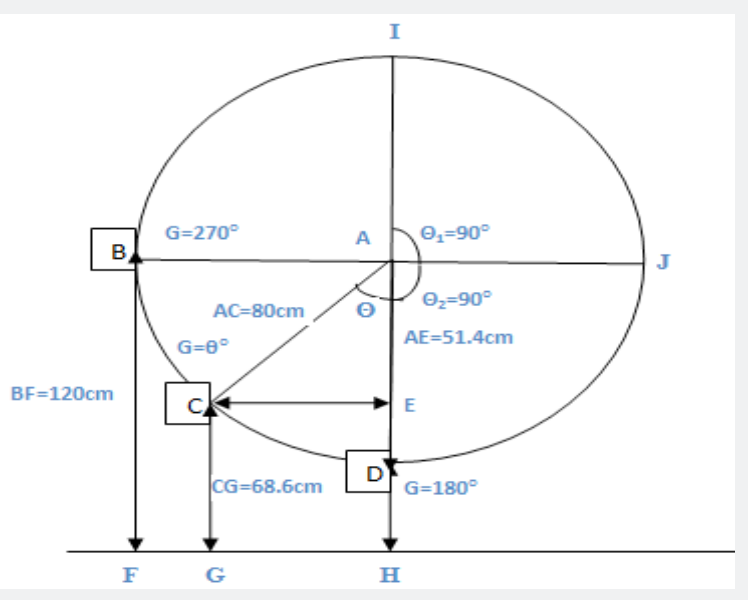

Figure 1: Describing the methodology used for the calculation of optimized gantry angle.

Table 2: Showing the derived gantry angles at respective heights of the source drawer flask from the floor.

\begin{tabular}{|c|c|c|}
\hline S. No. & $\begin{array}{c}\text { Height of Source Drawer Flask } \\
\text { from Floor }\end{array}$ & $\begin{array}{c}\text { Calculated Gantry } \\
\text { Angle }\end{array}$ \\
\hline 1. & $68.6 \mathrm{~cm}$ & $230.02^{0}$ \\
\hline 2. & $68.5 \mathrm{~cm}$ & $229.93^{0}$ \\
\hline 3. & $68.7 \mathrm{~cm}$ & $230.11^{0}$ \\
\hline
\end{tabular}

At the distance values of the center of the source drawer flask to floor, the derived values of Gantry angle are shown in table 2. The SD error is 0.10 The Gantry motion tolerance suggested by the manufacturers was 0.20 . Hence the optimized Gantry angle for the current source loading operation was $230^{\circ}$. The
$\mathrm{AE}=(\mathrm{AH}-\mathrm{EH})$

$(\mathrm{EH} \sim \mathrm{CG}=68.6 \mathrm{~cm})$

$\mathrm{AE}=(120 \mathrm{~cm}-68.6 \mathrm{~cm})$

$\mathrm{AE}=51.4 \mathrm{~cm}, \mathrm{AC}=80 \mathrm{~cm}$

$\operatorname{In}^{\Delta} \mathrm{ACE}$

$\operatorname{Cos} \theta=\mathrm{AE} / \mathrm{CA}$

$\operatorname{Cos} \theta=(51.4) / 80$

$\Theta=\cos ^{-1}(0.6425)$

$\Theta=50.020$ (Approx. 500)

Finally we got the optimized gantry angle i.e. $(\theta 1+\theta 2+\theta) 0=$ $\left(90^{\circ}+90^{\circ}+50^{\circ}\right)=230^{0}$ total time taken for the procedure comprising of 4 attempts was 1hour 50minutes out of which, the $4^{\text {th }}$ attempt made at Gantry $\theta$ $=230.1 \mathrm{o}$ took 7 minutes only.

\section{Discussion}

The review of literature showed limited material regarding detailed guidelines for source loading in teletherapy units. The extra time taken and also causing Radiation exposure by carrying out the 'Hit \& Trial' method based on working experience by service engineers at the time of source loading warrants a rigid protocol for source loading to be adopted. The result of this study documents that by following a rigid protocol, the number of trials undertaken for the cobalt- 60 source loading could have been reduced. In this exercise from 4 to 1 (75\%), overall time taken can also be reduced from 120 minutes to 20 minutes and thus also limiting the radiation exposure to all personnel involved; thereby making this procedure more friendly as per the ALARA principle.

\section{Conclusion}

A Standard operating Procedure can be recommended based on the above exercise. Following recommendation is being made:

The 'Standard Operating Procedure' to be observed is:

i. $\quad$ All civil works of the therapy room including the CCTV \& Air conditioning to be complete. The floor surface should be flat.

ii. The electrical and mechanical acceptance tests of the therapy unit should be carried out and be within their tolerance limits. 
iii. Gamma Zone monitor, emergency switches \& Survey meter should be in working condition.

iv. Personal Monitoring Devices and pocket dosimeters to be worn by the involved persons.

v. The gantry angle to be derived as per the calculations mentioned above.

vi. The planned procedure to be executed with apt attention and under minimal disturbances. vii. Strict record of various steps to be maintained.

\section{References}

1. Sahani G, Kumar M, Dash Sharma PK, et al. Compliance of Bhabhatron-II telecobalt unit with IEC standard - Radiation safety. J Appl Clin Med Phys 10(2): 2963.

2. BARC Bhabhatron-I telecobalt machine. Nuclear India 39(1-2): 1-2.

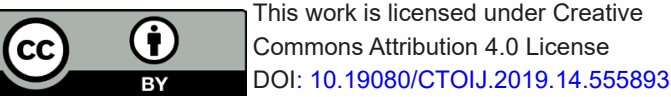

